\title{
HIDROLOGIA COMPARATIVA EN LA BOCA DE DOS SISTEMAS ANTIESTUARINOS DE BAJA CALIFORNIA DURANTE \\ OTOÑO E INVIERNO.
}

$$
\text { por }
$$

Salvador Galindo Bect

\author{
Bernardo P. Flores Baez \\ Instituto de Investigaciones Oceanológicas \\ Apartado Posta1 453 \\ Ensenada, Baja California, México.
}

RESUMEN

Se realizaron cuatro variaciones diurnas, dos en la boca de Bahía San Quintîn y dos en la del Estero de Punta Banda, que abarcaron otoño e invierno. En cuanto a las condiciones internas de los sistemas, el calentamiento por irradiación solar y la evaporación producida principalmente por los vientos, regulan los gradientes verticales de temperatura y de salinidad, produciendo inversiones en el primero de estos, aunque esta puede ser fácilmente destruída debido a la pequeña diferencia entre los valores de superficie y fondo, asi como al delgado espesor de la capa de agua y a las corrientes y turbulencias que se presentan.

ABSTRACT

Four diurnal variations were observed, two in the mouth of San Quintin Bay and two in the Estero de Punta Banda. These observations were taken during Autumn and Winter. Respect to the internal conditions of the systems, it has been found that heating due to solar radiation and evaporation produced principally by winds regulate the vertical gradients of temperature and salinity. These processes produce inversions in San Quintin Bay although this can be easily destroyed due to the small defferences between superficial and deep values as well as the shallow water layer and the currents and turbulence that are present. 


\section{INTRODUCCION}

Las lagunas costeras, esteros y estuarios son cuerpos de agua litoral semiprotegidos, de vital importancia desde el punto de vista biologico, ya que estos presentan una trampa nutricia, importante en el desarrollo de algunas especies de amplio espectro ecológico. El conocimiento de estas áreas altamente productivas implica el tratar de preservarlas, con la finalidad de introducir en estos cuerpos de agua, organismos que, localizados en los niveles tróficos iniciales, consuman esta alta productividad.

Los esfuerzos realizados para el conocimiento de estas lagunas costeras se han 1levado a cabo mediante objetivos a largo y corto plazo, por el Instituto de Investigaciones Oceanológicas de la Universidad Autónoma de Baja California (UABC) y el Centro de Investigación Científica y de Educación Superior de Ensenada (CICESE). Dada la importancia de las lagunas costeras, en la Bahia San Quintín y el Estero de Punta Banda, se han venido desarrollando desde hace algunos años estudios en diferentes áreas del conocimiento, como son Física, Química y Biología, todos tendientes a proporcionar una infraestructura científica que puede ser utilizada para el desarro110 de maricultivos (Acosta Ruiz y Alvarez Borrego, 1974, 1977; Lara Lara y Alvarez Borrego, 1975; Alvarez Borrego y Chee Barragán, 1976).

\section{OBJETIVO}

En el presente trabajo se exponen y se discuten los resultados de las mediciones de temperatura, salinidad y oxígeno disuelto obtenidos durante otoño e invierno de 1975, en 1a boca de dos sistemas antiestuarinos, Bahla San Quintín y Estero de Punta Banda, con el objeto de conocer la hidrología de estos dos sistemas y los mecanismos de intercambio entre el interior y exterior de los mismos.

\section{DESCRIPCION DEL AREA}

La Bahía de San Quintín se encuentra localizada en la costa noroccidental de Baja California, entre las latitudes $30^{\circ} 24^{\circ}$ y $30^{\circ} 30^{\prime} \mathrm{N}$ y entre las longitudes $115^{\circ} 57^{\prime}$ y $116^{\circ} 01^{\prime} \mathrm{W}$, aproximadamente a unos $200 \mathrm{~km}$ al sur de Ensenada, B.C. (Fig. 1). En el mar abierto inmediatamente al sur de la entrada de: la bahía, ocurren surgencias intensas (Dawson, 1951). La boca tiene poco más de un kilómetro de ancho (Chávez de Nishikawa y Alvarez Borrego, 1974). Esta bahía ha sido ampliamente descrita por Menzies (1962); Barnard, (1962, 1964 y 1970); Gorsline y Stewart, (1962); Dawson, (1951); Keen, (1962); Reish, (1963); Alvarez Borrego, Ballesteros Grijalva y Chee Barragán, (1975); Alvarez Borrego, Acosta Ruíz y Lara Lara, (1977). 
La Bahía de Todos Santos se encuentra localizada entre las latitudes $31^{\circ} 40^{\prime \prime}$ y $31^{\circ} 56^{\prime} \mathrm{N}$ y entre las longitudes $116^{\circ}$ $36^{\prime}$ y $116^{\circ} 50^{\prime} \mathrm{W}$ (Fig. 2). Es una bahía amplia y abierta al océano, con un área aproximada de noventa millas cuadradas.

E1 Estero de Punta Banda es una laguna costera localizada a lo largo del márgen sureste de la Bahía de Todos Santos, está separada de la bahía por una barra arenosa de poco más de $7 \mathrm{~km}$ de longitud, que parte de la base de Punta Banda hacia el noroeste. Esta Bahía ha sido ampliamente descrita por Walton, (1955); Benson, (1959); O'Brien y Zeevaert, (1968); Acosta Ruíz y Alvarez Borrego, (1974); Alvarez Borrego, Acosta Ruiz y Lara Lara, (1977); Pritchard et al, (1978).

\section{MATERIALES Y METODOS}

Se realizaron cuatro variaciones diurnas, dos en la boca de Bahía San Quintín y dos en la del Estero de Punta Banda, abarcando otoño e invierno. Los muestreos para Bahía San Quintín se 1levaron a cabo el 26-27 de noviembre de 1975 y $18-$ 19 de febrero de 1976. Para e1 Estero de Punta Banda el 17-18 de diciembre de 1975 y 24-25 de febrero de 1976. Se tomaron muestras de agua en la superficie y un metro arriba del fondo, a cada hora y durante un período de 24 horas, determinándose la temperatura del agua, salinidad y oxígeno disuelto, además se midieron las variables meteorológicas: temperatura ambiental $y$ velocidad del viento.

\section{RESULTADOS}

\section{Temperatura.}

Durante otoño e invierno en Bahía San Quintín, el ciclo de variación diurna de temperatura y salinidad presentó un comportamiento en relación inversa respecto a la fluctuación de la marea predicha para la localidad (Fig.s 3, 5, 15 y 17). En otoño se observó una inversión en el gradiente de temperatura durante la mayor parte del muestreo, con los valores mayores en el fondo, mientras que en invierno esta no fué tan marcada. Lo anterior también fué observado en el verano del mismo año por Alvarez Borrego et a1., (1977).

Para otoño en el Estero de Punta Banda, la temperatura presentó un comportamiento en relación Airecta respecto a la marea (Figs. 4 y 16). En el muestreo de invierno se observó una gran homogeneidad en la columna de agua con pequeñas inversiones en el gradiente. Se hace notar un cambio brusco ocurrido a las 02:00 horas en que la temperatura descendio hasta $10.4^{\circ} \mathrm{C}$ (Fig. 6). 


\section{Salinidad}

En otoño, la salinidad de Bahía San Quintín presentó al igual que 1a temperatura, una relación inversa respecto a la marea, además de un gradiente con los valores de fondo ligeramente mayores que 1 os superficiales (Figs. 7 y 15).

En invierno presentó dos inversiones de gradiente de salinidad a las 13:00 y 18:00 horas, las cuales coinciden con las de temperatura, 10 que puede implicar un cambio en el gradiente de densidad (Figs. 5 y 9).

En otoño, el Estero de Punta Banda presentô un comportamiento irregular con cambios bruscos en la salinidad, contrario a lo sucedido en invierno donde se observó homogeneidad (Figs. 8 y 10).

\section{Oxígeno}

En los muestreos de otoño e invierno la concentración de oxígeno disuelto en Bahía San Quintín presentó un comportamiento en relaciôn inversa respecto a la marea, con excepción de un descenso brusco ocurrido en otoño con valores mínimos de $3.35 \mathrm{ml} / 1$ en 1 a superficie y $1.79 \mathrm{ml} / 1$ en el fondo a las 17:00 horas (Figs. 11, 13, 15 y 17).

En otoño el Estero de Punta Banda observó una gran cantidad de cambios bruscos a través de todo el muestreo, con el gradiente invertido en la mayor parte del ciclo, contrario a lo sucedido en invierno (Figs. 12 y 14).

Velocidad del viento, Temperatura Ambiental y Marea.

La velocidad del viento en Bahía San Quintín, tanto en otoño como en invierno, varió en un rango de 2.0 a 12.0 $\mathrm{m} / \mathrm{seg}$, mientras que la temperatura ambiental entre los $13.0 \mathrm{y}$ $16.0^{\circ} \mathrm{C}$ (Figs. 19 y 21), haciéndose notar que los valores máximos para las dos observaciones se registraron entre las 13:00 $y$ las 16:00 horas.

E1 Estero de Punta Banda presentó vientos de menor intensidad que Bahía San Quintín, con un rango de 0.0 a 3.0 $\mathrm{m} / \mathrm{seg}$ en otoño y $0.0 \mathrm{a} 9.0 \mathrm{~m} / \mathrm{seg}$ en invierno (Figs. 20 y 22).

Las curvas de marea presentadas en este trabajo, corresponden a la marea predicha para la localidad ya que no se efectuaron mediciones directas (Figs. 15. 16, 17 y 18). 
GALINDO-FLORES

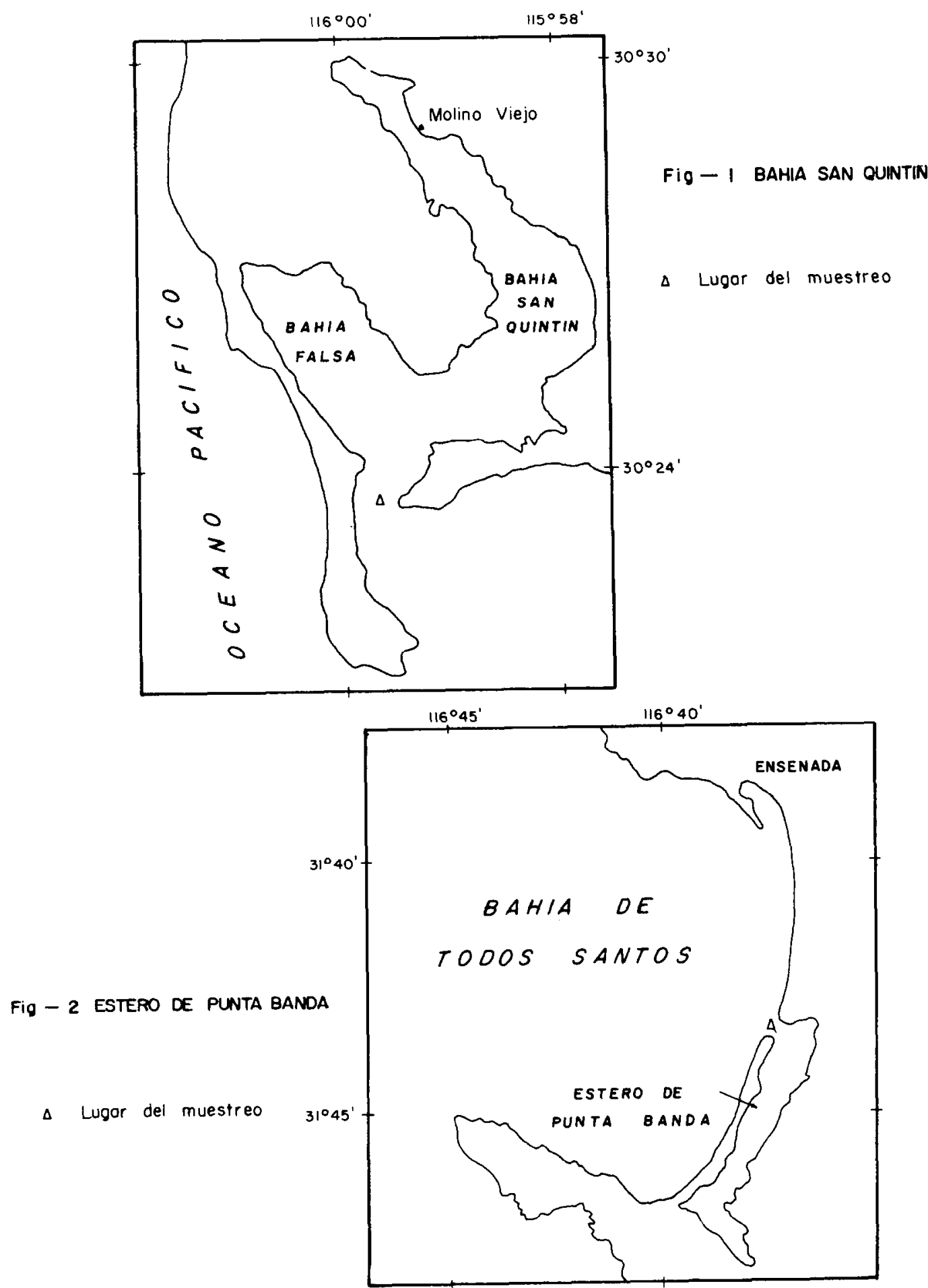




\section{HIDROLOGIA COMPARATIVA EN DOS ANTIESTUARIOS}
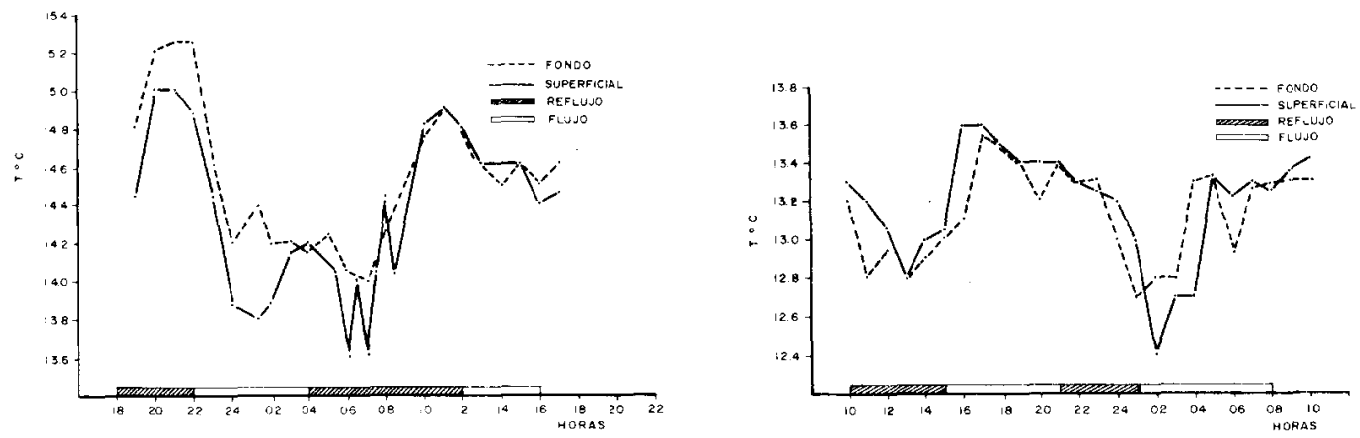

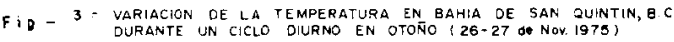

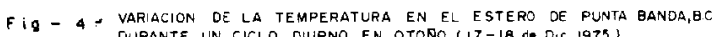

DURANTE UN CICLO DILRNO EN OTONO $(17-18$ de Dic. 1975$)$
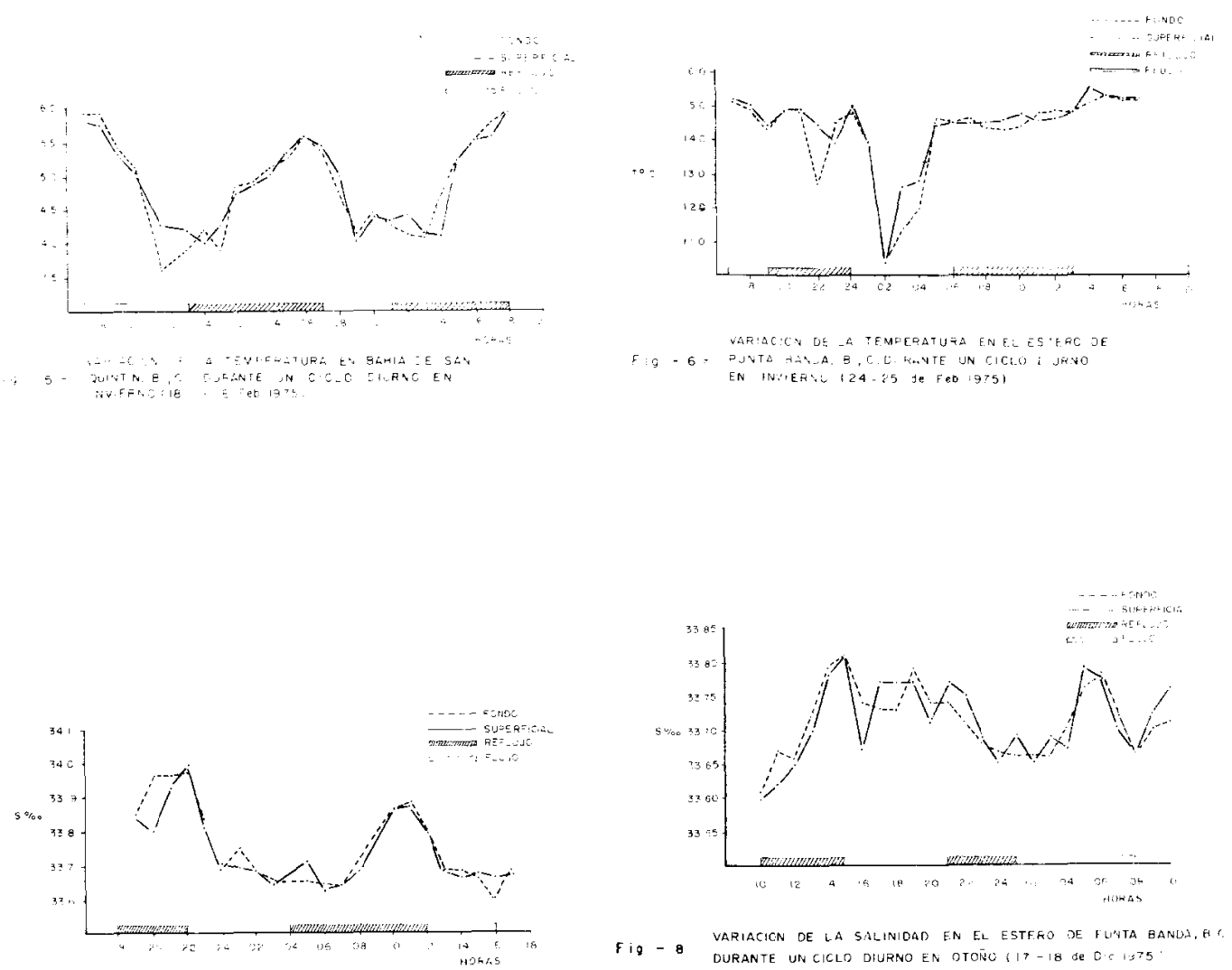

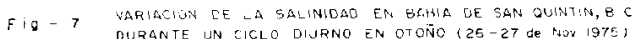




\section{GAL INDO-FLORES}

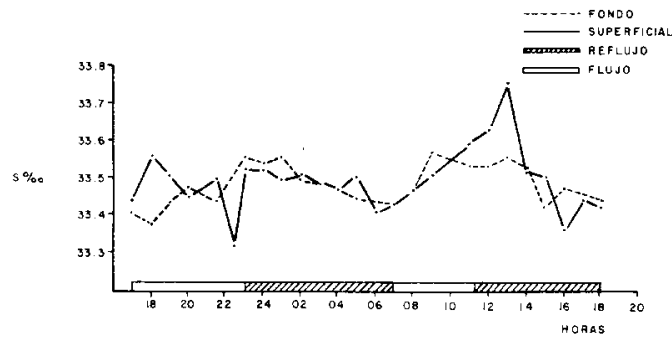

VARIACION DE LA SALINIDAD EN BAHIA DE SAN OUINTIN B. C. DURANTE
DE FE. $(976)$

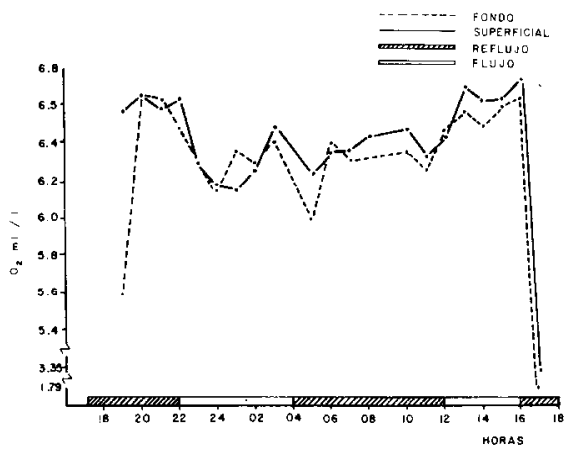

VARIACION DEL OXIGENO DISUELYO EN BAHIA DE SAN QUINTIN, B, C. DURANTE UN CICLO DIURNO EN OTOANO FIO- 11 QUINTIN, B, C. DURANTE
(26-27 de Nov $(975)$

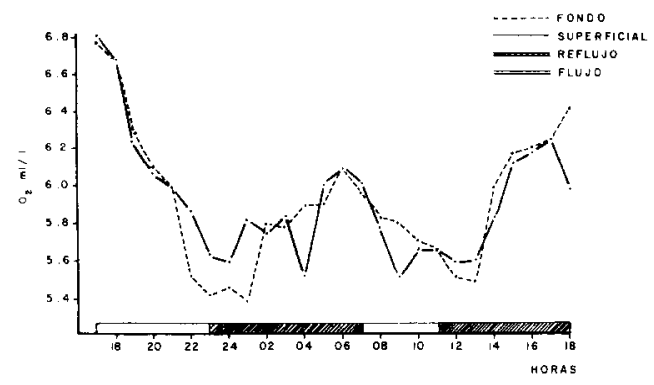

$\mathrm{F}: 0-13$ OUINTIN, B, C DURANTE UN CICLO OIURNO EN INVIERNo $(18-19$ de Dic. 1976$)$

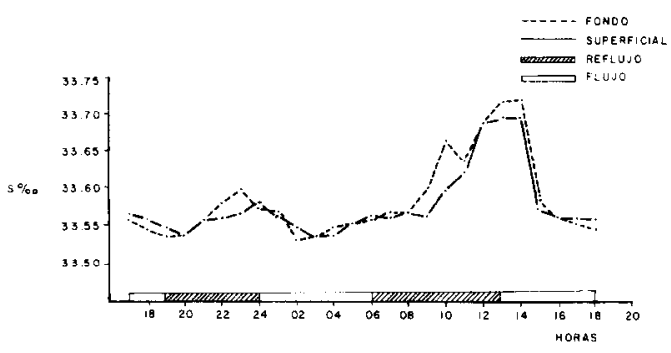

VARIACION OE LA SALINIDAO EN EL ESTEROD DE PUNTA FIg- 10 BANDA B., C DURANTE UN GIGLO DIURNO EN INVIERNO

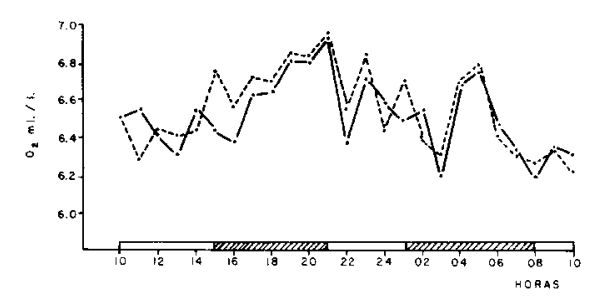

VARIACION DEL OXIGENO DISUELTO EN ESTERO DE PUNTA BANDA, B.,C DURANTE UN CICLO DIURNO EN OTOÑO $(17-18$ de Dic. 1976$)$.

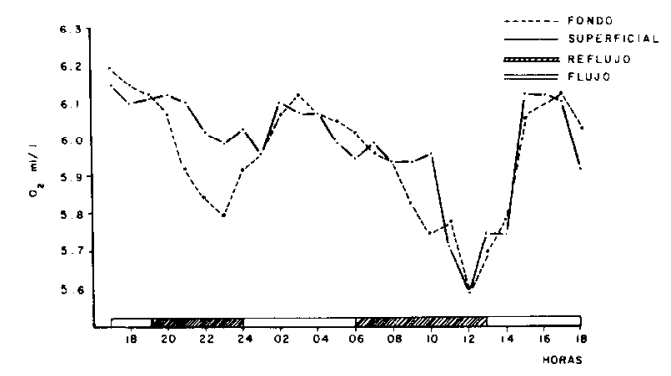

VARIACION DEL OXIGENO Disuelto EN ESTERo oE PUNTA BANDA, B, C DURANTE UN CICLO DIURNO EN INVIERNO(24-25 de Feb 1976$)$. 

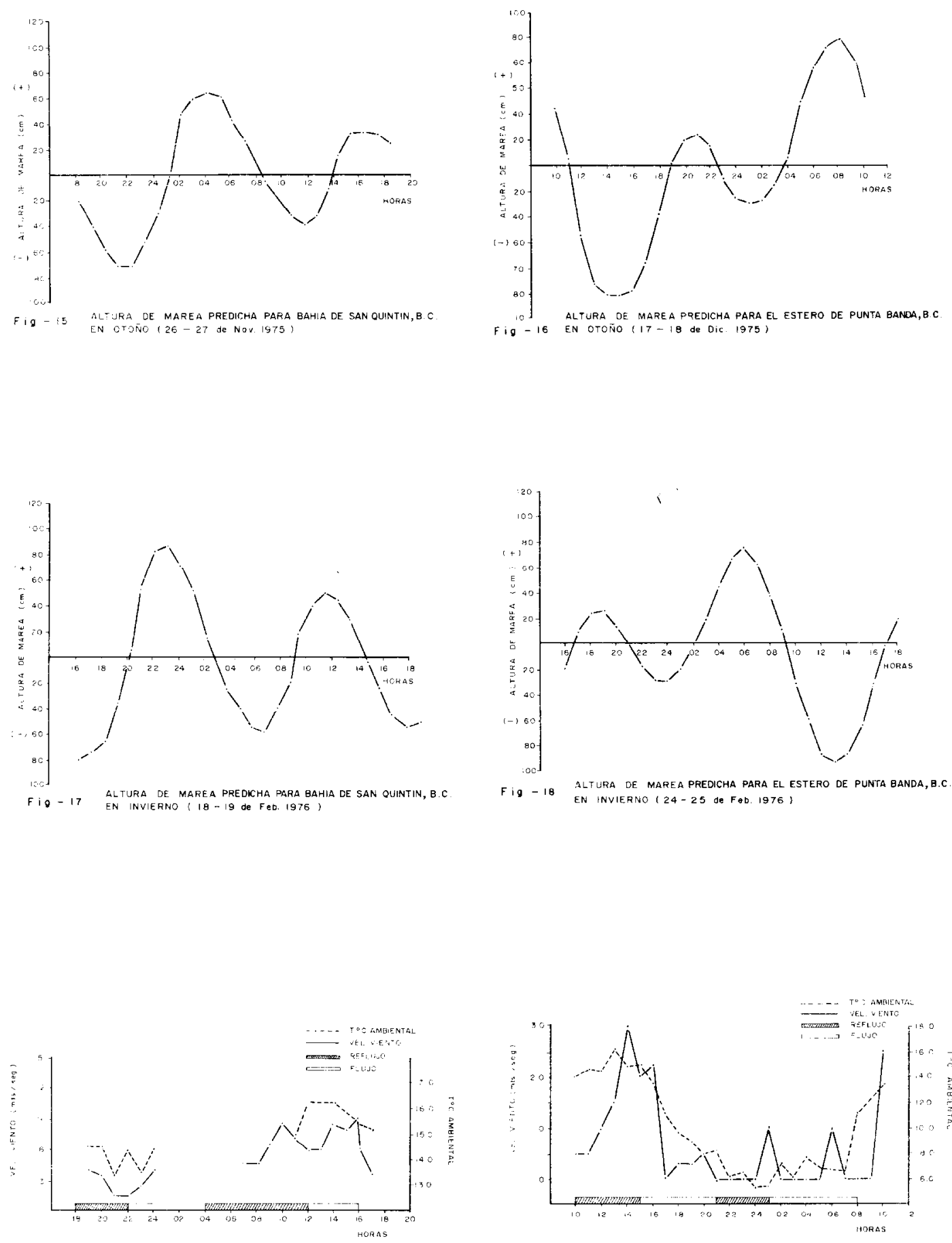

FIg- I V VARIACION OE LA TEMPERATURA AMBIENTAL Y VELOCIDAD OEL VIENTO ELA BAHIA DE SAN OLINTIN, B C OURANT
CICLO DIUPNO EN OTONOO $126-27$ de NOY. 1975 I

VARIACION DE LA TEMPERATURA AMBIENTAL Y VELOCIOAD

Fig - 20 DEL VIENTO EN EL ESTERO DE PUNTA BANOA, B.C. DURANTE UN CICLO DIURNO EN OTOÑO $(17-18$ de DIE 1975) 

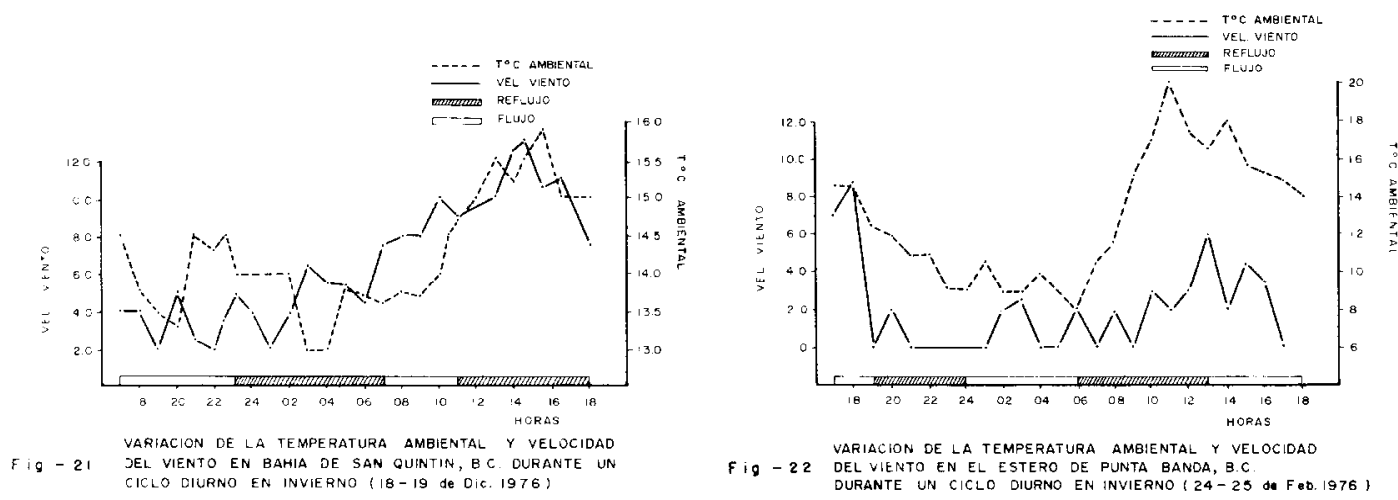

\section{DISCUSIONES}

La representatividad de los resultados obtenidos en el presente estudio es muy limitada, ya que las mediciones se hicieron durante un día de cada estación del año, pudiendo explicarse algunos procesos de intercambio entre el interior y el exterior de estos cuerpos de agua.

Bahía San Quintín en otoño e invierno refleja la influencia de surgencias cercanas a la boca de la bahía (Dawson, 1951), condiciones turbulentas propias de la boca y aguas de diferentes propiedades provenientes del interior del sistema; durante este período la bahía aporta oxígeno al océano adyacente. Debe hacerse notar, sin embargo, que las mediciones corresponden a un sólo día y que las condiciones de surgencia no son permanentes y prevalecen en verano.

Los altos valores de temperatura y salinidad detectados en el reflujo, demuestran que estos tienden a incrementar de la boca a la cabeza de la laguna (Chávez de Nishikawa y Alvarez Borrego, 1974). Los pequeños incrementos de temperatura y salinidad en la región interna con vientos débiles, pueden invertir el gradiente de temperatura (Postma, 1965; Alvarez Borrego et al, (1977). Si la estratificación es desarrollada, las aguas con mayor temperatura y salinidad se encontrarán cerca del fondo, de tal manera que durante el reflujo se observará un transporte de agua más densa a lo largo del fondo del canal hacia el mar abierto (Groen, 1969; Pritchard et al, 1978). Sin embargo, esto se marca claramente en el muestreo de otoño, donde el agua en el interior de la bahía ha sufrido un incremento en la salinidad, prevaleciendo sobre la temperatura, más no así en invierno, donde la inversión del gradiente de la temperatura es contrabalanceada por la escasa evaporación, hacién- 
do que tal estratificaciôn inversa, sea fácilmente destruída por las corrientes, pudiendo incluso ocurrir homogeneidad vertical. Postma (1965), encontró que las diferencias de densidad entre la baja y alta marea fueron muy pequeñas (menores de $0.001)$, pero aûn asî existen.

Bahía San Quintín se encuentra cubierta en su mayor parte por "pastos marinos" (Zostera sp), los cuales aportan "detritus orgánico" formando zonas de reducción. Lo anterior viene a ser corroborado por los bajos valores de oxígeno disuelto detectados en otoño durante el reflujo.

Contrario a lo que sucedió durante primavera y verano de 1975, en que los valores de temperatura más bajos se presentaron en la boca de Bahía San Quintín (Alvarez Borrego et al, 1977), en el presente trabajo se registraron en la boca del Estero de Punta Banda, ya que la temperatura ambiental en esta región fué menor.

E1 aumento del oxígeno disuelto durante el reflujo es producido por el intenso oleaje que comúnmente se presenta en la boca del estero (Alvarez Borrego et al, 1977), mientras que en el reflujo descendieron los valores, debido probablemente a procesos de oxidación y respiración durante la permanencia de las aguas en el interior del sistema, denotando las condiciones internas y externas.

En invierno se hizo evidente un cambio brusco ocurrido a las dos de la mañana, debido posiblemente a una corriente paralela a la costa con dirección norte detectada por Cabrera Muro (1974) y Contreras Rivas (1973). Alvarez Borrego et al (1977), encontraron un cambio similar en 1a época de primavera atribuyéndola a este tipo de corrientes. Las bajas temperaturas registradas corroboran 10 observado por Morales Zúñiga (1977) y Argote Espinoza (citado por Morales Zúñiga, 1977).

Contrario a 1o ocurrido en Bahia San Quintín, e1 Estero de Punta Banda presentó una inversión en el gradiente de oxígeno disuelto con los valores de fondo mayores que los superficiales como consecuencia de las bejas temperaturas que provocan una alta solubilidad.

\section{CONCLUSION}

Respecto a las condiciones internas de los sistemas, el calentamiento por irradación solar y la evapcoración producida principalmente por los vientos, regulan los gradientes verticales de temperatura y salinidad, pudiendo producir inversiones en el primero de éstos, aunque esta inversión puede ser fácilmente destruída por las corrientes y turbulencias, debido al débil gradiente vertical, así como al delgado espesor de la capa de agua. 
BIBLIOGRAF IA

ACOSTA RUIZ, M. de J. y S. Alvarez Borrego, 1974. Distribuciôn superficial de algunos parámetros hidrológicos, físicos y químicos en el Estero de Punta Banda, B.C., en otoño e invierno. Ciencias Marinas, 1(1):16-45.

ALVAREZ BORREGo, S., M. de J. Acosta Ruiz y J.R. Lara Lara, 1977. Hidrología comparativa de las bocas de dos antiestuarios de Baja California. Ciencias Marinas, 4(1):1-11.

ALVAREZ BORREGO, S., G. Ballesteros Grijalva y A. Chee Barragán, 1975. Hidrología de la Bahía San Quintín, B.C., en verano, otoño e invierno. Ciencias Marinas, 2(2):19.

ALVAREZ BORREGO, S. y A. Chee Barragán, 1976. Distribución superficial de fosfatos y silicatos en Bahía de San Quintin, B.C. Ciencias Marinas, 3(1):51-61.

BARNARD, L., 1962. Benthic marine explotation of Bahla de San. Quintin, B.C. 1960-1961. Pacific Naturalist, 3:249274.

--_-----, 1964. Marine Amphipoda of Bahia San Quintin, B.C. Pacific Naturalist, 4:55-139.

--------, 1970. Benthic ecology of Bahia San Quintin, B.C. Smithsonian Contribution to Zoology, No. 44.

BENSON, R.H., 1959. Ecology of recent ostracods of the Todos Santos Bay Region, B.C., Mexico. The University of Kansas Paleontological Contributions. Arthropods 1: $1-80$.

CABRERA MURO, H., 1974. Distribución de temperatura en la Bahía de Todos Santos. Ciencias Marinas, 1(1):65-77.

CONTRERAS RIVAS, I., 1973. Influencia termohalina de las aguas del Estero de Punta Banda en la Bahía de Todos Santos, B.C. Tésis Profesional. Escuela Superior de Ciencias Marinas, Universidad Autónoma de Baja California.

CHAVEZ de NISHIKAWA, A.G. y S. Alvarez Borrego, 1974. Hidrología de la Bahía de San Quintín, B.C., en invierno y primavera. Ciencias Marinas, 1(2):31-62.

DAWSON, E.Y., 1951. A Further Study of Upwelling and Vegetation Along Pacific Baja California, Mexico. Journal of Marine Research, 10(1):39-58.

GORSLINE, D.I.F. y R.A. Stewart, 1962. Benthic Marine exploration of Bahia San Quintin, B.C., 1960-61. Marine and Quaternary Geology. Pacific Naturalist, 2:283-319. 
GROEN, P., 1969. Physical Hydrology of Coastal Lagoons. Lagunas Costeras, un simposio. Mem. Simp. Intern. Lagunas Costeras. UNAM-UNESCO, Nov. 28-30, 1967. México, D.F. 275-280.

KEEN, A.M., 1962. A new west Mexican subgenus and new species of Montacutidae (Mollusca:Pelecypoda), with a list of Mollusca from Bahia de San Quintin. Pacific Naturalist, 3:321-328.

LARA LARA, J.R. y S. Alvarez Borrego, 1975. Ciclo anual de Clorofilas y Producción Orgánica Primaria en Bahía de San Quintín, B.C. Ciencias Marinas, 1(1):77-97.

MENZIES, R.J., 1962. The Marine Isopod Fauna of Bahia San Quintin, B.C., Mexico. Pacific Naturalist, 3:337-348.

MORALES ZUÑIGA, C., 1977. Variaciones estacionales de la temperatura en 1a Bahía de Todos Santos, B.C. Ciencias Marinas, 4(1):23-33.

O'BRIEN, M.P. y L. Zeevaert, 1968. Design of a small tidal inlet. Proceedings of the eleventh Conference on Coastal Engineering. London, England.

POSTMA, H., 1965. Water circulation and suspended matter in B.C. Lagoons. Netherlands Jour. Sea Res. 2:566-604.

PRITCHARD, D.W., R. de la Paz, H. Cabrera, S. Farreras y E. Morales, 1978. Hidrografía Física del Estero de Punta Banda, B.C. Ciencias Marinas, 5(2):1-23.

REISH. D.J., 1963. A quantitative study of the Benthic Polychaetus annelids of Bahia San Quintin, B.C. Pacific Naturalist, 3:399-436.

WALTON, W.R., 1955. Ecology of Livin Benthonic Foraminifera, Todos Santos Bay, Baja California. J. of Paleontology, Vol. 29, No. 6, 952-1018. 